\title{
NMR-based metabolomics identifies patients at high risk of death within two years after acute myocardial infarction in the AMI-Florence II cohort
}

Alessia Vignoli ${ }^{1,2}$, Leonardo Tenori ${ }^{1,3}$, Betti Giusti ${ }^{3,4^{*}}$ (D), Panteleimon G. Takis ${ }^{5}$, Serafina Valente ${ }^{4}$, Nazario Carrabba ${ }^{4}$, Daniela Balzi ${ }^{6}$, Alessandro Barchielli ${ }^{6}$, Niccolò Marchionni ${ }^{3,4}$, Gian Franco Gensini ${ }^{7}$, Rossella Marcucci ${ }^{3,4}$,

Claudio Luchinat ${ }^{1,2,8}$ and Anna Maria Gori ${ }^{3,4}$

\begin{abstract}
Background: Risk stratification and management of acute myocardial infarction patients continue to be challenging despite considerable efforts made in the last decades by many clinicians and researchers. The aim of this study was to investigate the metabolomic fingerprint of acute myocardial infarction using nuclear magnetic resonance spectroscopy on patient serum samples and to evaluate the possible role of metabolomics in the prognostic stratification of acute myocardial infarction patients.
\end{abstract}

Methods: In total, 978 acute myocardial infarction patients were enrolled in this study; of these, 146 died and 832 survived during 2 years of follow-up after the acute myocardial infarction. Serum samples were analyzed via highresolution ${ }^{1} \mathrm{H}$-nuclear magnetic resonance spectroscopy and the spectra were used to characterize the metabolic fingerprint of patients. Multivariate statistics were used to create a prognostic model for the prediction of death within 2 years after the cardiovascular event.

Results: In the training set, metabolomics showed significant differential clustering of the two outcomes cohorts. A prognostic risk model predicted death with 76.9\% sensitivity, 79.5\% specificity, and 78.2\% accuracy, and an area under the receiver operating characteristics curve of 0.859 . These results were reproduced in the validation set, obtaining $72.6 \%$ sensitivity, $72.6 \%$ specificity, and $72.6 \%$ accuracy. Cox models were used to compare the known prognostic factors (for example, Global Registry of Acute Coronary Events score, age, sex, Killip class) with the metabolomic random forest risk score. In the univariate analysis, many prognostic factors were statistically associated with the outcomes; among them, the random forest score calculated from the nuclear magnetic resonance data showed a statistically relevant hazard ratio of $6.45\left(p=2.16 \times 10^{-16}\right)$. Moreover, in the multivariate regression only age, dyslipidemia, previous cerebrovascular disease, Killip class, and random forest score remained statistically significant, demonstrating their independence from the other variables.

(Continued on next page)

\footnotetext{
* Correspondence: betti.giusti@unifi.it

Alessia Vignoli and Leonardo Tenori are Contributed equally

Claudio Luchinat and Anna Maria Gori are Share senior authorship

${ }^{3}$ Department of Experimental and Clinical Medicine, University of Florence,

Florence, Italy

${ }^{4}$ Careggi Hospital, Florence, Italy

Full list of author information is available at the end of the article
}

(c) The Author(s). 2019 Open Access This article is distributed under the terms of the Creative Commons Attribution 4.0 International License (http://creativecommons.org/licenses/by/4.0/), which permits unrestricted use, distribution, and reproduction in any medium, provided you give appropriate credit to the original author(s) and the source, provide a link to the Creative Commons license, and indicate if changes were made. The Creative Commons Public Domain Dedication waiver (http://creativecommons.org/publicdomain/zero/1.0/) applies to the data made available in this article, unless otherwise stated. 
(Continued from previous page)

Conclusions: For the first time, metabolomic profiling technologies were used to discriminate between patients with different outcomes after an acute myocardial infarction. These technologies seem to be a valid and accurate addition to standard stratification based on clinical and biohumoral parameters.

Keywords: Acute myocardial infarction, Nuclear magnetic resonance, Serum, Metabolomics, Biomarker, Prognosis, Precision medicine

\section{Background}

Among cardiovascular diseases (CVDs), acute coronary syndrome (ACS) represents the most common cause of emergency hospital admission and it is associated with the highest mortality and morbidity $[1,2]$. The prognosis is directly associated with timely initiation of revascularization, and misdiagnosis or late diagnosis may have unfavorable clinical implications. Established risk stratification tools such as the Global Registry of Acute Coronary Events (GRACE) and the Thrombolysis In Myocardial Infarction risk scores are derived from demographic, clinical, laboratory, and electrocardiogram-related variables [3, 4]. These do not incorporate the use of newer biomarkers, which could represent different pathophysiologic processes and provide complementary prognostic information, thereby improving risk stratification beyond traditionally used variables.

Several studies have evaluated the potential clinical usefulness of new biomarkers able to identify patients who had a poor outcome. In particular, high levels of inflammatory markers such as C-reactive protein and interleukin-8 had long-term prognostic utility in patients with ACS that undergone coronary revascularization [5, 6]. However, no conclusive and consistent data about the prognostic utility of measuring inflammatory markers in the early phase of ACS are available in the literature.

A number of studies have evidenced that a global approach, such as genomics, proteomics, or metabolomics, may represent a valid strategy for improving current knowledge about pathophysiological mechanisms and for identifying ACS patients at high risk of secondary atherothrombotic events or premature death.

Metabolomics is the accepted name for the -omic science that deals with the characterization of the metabolome, in turn defined as the whole set of metabolites in a certain biological system, such as a cell, tissue, organ, or entire organism [7]. The two leading analytical techniques used to perform metabolomics are mass spectrometry (MS) and nuclear magnetic resonance (NMR) spectroscopy. Both techniques yield information about many different molecules in a single measurement, and can be used to determine structures and concentrations of metabolites [8]. Nevertheless, each technique has its own strengths and limitations. MS overshadows NMR in terms of numbers of compounds resolved (of the order of $\left.10^{3}[9]\right)$, with a sensitivity down to the picomolar and requiring a very small volume of the biospecimen; however, reproducibility is still a limitation of MS, which must be overcome by an extensive and time-consuming use of standards and quality control samples. NMR analysis is high-throughput [7], and NMR data are highly reproducible [10] and intrinsically quantitative over a wide dynamic range, as demonstrated by numerous ring trials performed by many different NMR laboratories [10]. NMR gives immediately qualitative and quantitative information on around $10^{2}$ different small molecules present in a biological sample [11], and has already provided a global picture of a wide range of metabolic processes underlying complex and multifactorial diseases such as ACS.

Recently, the metabolomic approach has been applied to identify a risk profile in heart failure patients [12-14], atrial fibrillation patients [15], and diabetic patients [16]. In the setting of ACS, studies have characterized the metabolic biosignature of myocardial ischemia [9, 17-20], identified altered signatures in lipid metabolism in patients with angina or myocardial infarction with respect to control subjects [21], and identified microbial metabolites in urine associated with coronary heart disease [22].

Risk stratification should identify individuals at high risk who require more intensive therapy, or, conversely, help avoid drug overuse and associated side effects in patients with a favorable prognosis. In this framework, the aim of the present study was to evaluate the impact of the metabolomic fingerprint on the occurrence of cardiovascular death in acute myocardial infarction (AMI) patients after percutaneous coronary intervention.

\section{Methods}

This study was part of a collaborative project between the Department of Medical and Surgical Critical Care of the University of Florence and the Magnetic Resonance Centre (CERM) of the University of Florence.

\section{Study population}

The study population comprised 978 out of 1496 patients admitted to the coronary units of the six hospitals (five community hospitals and one university hospital, the Careggi Hospital) of the Florence health district 
between April 2008 and April 2009, and enrolled in the frame of the Florence Acute Myocardial Infarction-2 (AMI-Florence 2) registry [23]. In the present study, we evaluated 978 patients (345 women and 633 men, median age 74 years); among them, 146 patients died within 2 years of the AMI event and 832 patients survived for at least 2 years. The 2-year vital status was assessed by consulting the registry office of the city of residence. Mortality analysis was therefore censored at 24 months after AMI or at date of death, whichever occurred earlier. For this study, 35\% of the AMI-Florence 2 population was excluded because of the lack of a good quality blood sample for the metabolomic analyses and/or because follow-up information was not available (Additional file 1: Figure S1). However, according to a standard power analysis [24] using a $t$ test as the test statistic, and fixing an alpha level of 0.05 for a significant comparison, it was found that having 146 patients who died and 832 patients who survived was enough to detect small to medium effects (Cohen's d $\sim 0.25$ ) with a statistical power of $80 \%$; furthermore, the training and validation sets separately showed adequate statistical power (Cohen's $d=0.54$ and 0.29 , respectively). Blood samples were collected 24-48 h after percutaneous coronary intervention (PCI) and overnight fasting. All information about inclusion criteria and treatment of the patients are detailed in Additional file 1: Supplementary material.

All subjects gave written informed consent. The study (number 11/2008) complies with the Declaration of Helsinki and was approved by the ethics committees of the local health unit, the University of Florence, and Careggi Hospital (19 March 2008).

\section{NMR analyses}

Samples were prepared following the standard protocols detailed by Bernini et al. [25]. According to standard practice [26], all spectra were acquired at $310 \mathrm{~K}$ using a Bruker $600 \mathrm{MHz}$ spectrometer (Bruker BioSpin), and for each serum sample three one-dimensional ${ }^{1} \mathrm{H}$-NMR spectra, namely nuclear Overhauser effect spectroscopy (NOESY), Carr-Purcell-Meiboom-Gill (CPMG), and Diffusion-edited spectra, were acquired, allowing the selective detection of different molecular weight metabolites. A detailed description of the sample preparation and experiments is presented in the Additional file 1: Supplementary material and Figure S1.

Each one-dimensional spectrum in the range $0.2-10.00$ ppm was segmented into $0.02 \mathrm{ppm}$ chemical shift bins and the corresponding spectral areas were integrated using AMIX software (version 3.8.4, Bruker BioSpin). The region between 4.5 and $5.0 \mathrm{ppm}$ containing the residual water signal was removed and the dimension of the system was reduced to 466 bins. The total spectral area was calculated on the remaining bins and total area normalization was carried out on the data prior to pattern recognition.

\section{Statistical analysis}

Data analyses were performed using the open source software R. For the demographic and baseline characteristics, the $t$ test was used for comparison between groups and the chi-square test for comparison between categorical variables.

For the multivariate data analyses of the NMR data, the group of 978 patients was randomly split into two independent cohorts [27]: a training set constituting 80 patients who survived and 40 who died, and a validation set constituting all remaining patients (106 patients who died and 752 who survived). A prospective power analysis [24] was employed to determine the minimum number of patients (surviving and dead) that would need to be retained in the training set to have a sufficiently powered model, thereby maintaining a large fraction of samples for the validation to guarantee a reliable estimation of the performance of the model. After determining that 80 patients who survived and 40 who died should be retained in the training set, the allocation between training and validation was performed randomly.

The initial analysis was restricted to the training set and the first step was to establish if serum metabolomic profiles could distinguish between patients who survived and died within 2 years after the cardiovascular event. For this purpose, a random forest (RF) classifier [28] was built (considering for each sample the full spectrum; thus, no choice of particular metabolite was performed). The percentage of trees that assign one sample to a specific class can be inferred as a probability of class belonging [29-31]. For each patient, a score was created that expressed the extent to which the serum metabolomic profile appeared to be similar to the profile of one of the patients who died, designated as the 'RF risk score'. For each patient, three RF scores were derived using the three types of spectra acquired. For all calculations, the $\mathrm{R}$ package 'Random Forest' [32] was used to grow a forest of 2,000 trees, using the default settings (see Additional file 1: Supplementary material for further details on the RF approach).

The next step was to test the hypothesis that a metabolomic signature similar to that of one of the patients who died would be predictive of death within 2 years after the cardiovascular event. Using receiver operating characteristics (ROC) analysis ("colAUC" function of the $\mathrm{R}$ package "caTools") and Harrell's c index ("cindex" function on the R package "dynpred"), the performances of the RF risk scores were compared with the actual outcome. To delineate high risk of death, a cut-off for the 
RF risk score was calculated in the training set that optimized accuracy, sensitivity, and specificity, and the performance of the model was subsequently tested in the validation set.

The performances of the NOESY RF score were evaluated by calculating a Cox proportional hazards regression model [33] using the function "coxph" (R package "Survival") and the model significance was assessed through a likelihood-ratio test and by calculating the model concordance. The independent prognostic capacity of the RF risk score model in comparison with standard prognostic features was also evaluated using Cox models. The performance of NOESY RF scores was also compared with the performance of the GRACE score and with a linear combination of the two scores through ROC analysis, Harrell's c index, and univariate Cox models.

The spectral regions related to 23 metabolites, present in concentrations above the detection limit $(>1 \mu \mathrm{M})$ in all samples (up to 30-40 different metabolites could be quantified in each sample [34]), were assigned in the CPMG NMR spectra by using matching routines of AMIX 3.8.4 (Bruker BioSpin) in combination with the BBIOREFCODE database (Bruker BioSpin) and the freely available Human Metabolome DataBase [35], and quantified. Metabolite quantification was determined by software developed in-house based on standard line-shape analysis methods. Using this approach, each NMR region of interest was decomposed and deconvoluted into its component parts that corresponded to its number of protons, and then integrated to obtain the metabolite concentrations in arbitrary units (Additional file 1: Figure S2). Wilcoxon signed-rank test [36] was chosen to infer differences between the metabolites concentrations of the outcome groups on the biological assumption that metabolite concentrations are not normally distributed, and false discovery rate correction was applied using the Benjamini-Hochberg method [37]. An adjusted $P$-value $<0.05$ was deemed significant. Effect size using Cliff's delta [38] was calculated by means of the R package "effsize".

The statistical approach described above was also used to build sex-specific statistical models based on NOESY spectra.

NMR and clinical data are freely available in the Open-Access Database Repository MetaboLights from October 2017 with the accession number MTBLS395 (http://www.ebi.ac.uk/metabolights).

\section{Results}

Demographic and clinical characteristics of the enrolled patients are shown in Table 1. The characteristics of patients according to gender are also reported in Additional file 1: Table S1.

\section{NMR spectra}

For each sample three NMR metabolomic profiles were obtained using NOESY, CPMG, and Diffusion-edited pulse sequences (Additional file 1: Figure S3). According to the peculiar characteristics of each NMR experiment, high and/or low molecular weight metabolites can be detected. An exploratory principal component analysis of the dataset is reported in Additional file 1: Figure S4.

\section{Discrimination of the outcomes and the RF scores in the training set}

The metabolomic profiles of 80 patients who survived and 40 who died (training set) were classified using the RF classifier. The profiles showed significant differential clustering, with good separation of the two groups using each type of NMR spectra: NOESY (Fig. 1a), CPMG, and Diffusion. Using ROC analyses, the areas under the curve (AUC) obtained were 0.859 for NOESY spectra (Fig. 1b), 0.857 for CPMG spectra, and 0.775 for Diffusion editing spectra. NOESY and CPMG models showed approximately the same performances, and the discrimination between the two outcome groups can be ascribed to both low (mostly) and high molecular weight metabolites. Thus, it was decided to use the NOESY spectra in the further analyses because they contained both high and low molecular weight metabolite information.

A threshold of $\geq 0.454$ for the NOESY RF score was set to optimize accuracy, sensitivity, and specificity; this optimized threshold yielded $76.9 \%$ (95\% confidence interval (CI) $76.5-77.3 \%)$ sensitivity, $79.5 \%$ (95\% CI 78.4-80.6\%) specificity, and 78.2\% (95\% CI 77.6-78.7\%) accuracy.

The goodness of fit of the NOESY RF model was calculated using a Cox proportional hazards regression model: NOESY RF showed a hazard ratio of 7.4 (95\% CI 3.5115.6) with a $p$-value of $3.45 \times 10^{-09}$ calculated using the likelihood-ratio test, and a concordance coefficient of 0.73 .

Moreover, the NOESY RF model was robust with respect to different strategies of model validation; indeed, a mean AUC of 0.805 (95\% CI 0.795-0.814) was obtained with 100 cycles of classical Monte Carlo cross-validation with data split 80 to $20 \%$ (training set, validation set). Furthermore, our approach was robust with respect to the origin of samples: only slight differences in the AUC were obtained when using samples from the Careggi University Hospital as the training set to build the RF model and samples from all the other hospitals as the validation set (and vice versa) (Additional file 1: Figure S5).

\section{Outcome prediction by NOESY RF score in a validation set of patients}

The validation set (106 patients who died and 752 who survived) was evaluated using an unsupervised analysis. 
Table 1 Demographic and clinical characteristics

\begin{tabular}{|c|c|c|c|}
\hline & Survived ( $n=832$ ) & Died $(n=146)$ & $p$-value \\
\hline \multicolumn{4}{|l|}{ Demographic characteristics, } \\
\hline Age (years), median (IQR) & $72(62-80)$ & $82(78-83)$ & $<2.20 \times 10^{-16}$ \\
\hline Female sex, $n(\%)$ & $278(33.4)$ & $67(45.9)$ & $4.86 \times 10^{-03}$ \\
\hline \multicolumn{4}{|l|}{ Cardiovascular risk factors, n (\%) } \\
\hline Hypertension & $537(64.5)$ & $104(71.2)$ & $1.21 \times 10^{-01}$ \\
\hline Dyslipidemia & $294(35.3)$ & $32(21.9)$ & $3.55 \times 10^{-03}$ \\
\hline Current smokers & $226(27.2)$ & $20(13.7)$ & $1.45 \times 10^{-04}$ \\
\hline Ex-smokers & $21(2.5)$ & $8(5.5)$ & $4.01 \times 10^{-01}$ \\
\hline CAD & $220(26.4)$ & $17(11.6)$ & $5.03 \times 10^{-04}$ \\
\hline Diabetes & $197(23.7)$ & $61(41.8)$ & $6.32 \times 10^{-06}$ \\
\hline \multicolumn{4}{|l|}{ Medical history, n (\%) } \\
\hline Myocardial infarction & $164(19.7)$ & $48(32.9)$ & $5.23 \times 10^{-04}$ \\
\hline Angina, onset $>1$ month & 119 (14.3) & $24(16.4)$ & $8.96 \times 10^{-01}$ \\
\hline Angina, onset $\leq 1$ month & 149 (17.9) & $15(10.3)$ & $3.78 \times 10^{-02}$ \\
\hline CABG & $41(4.9)$ & $10(6.8)$ & $4.39 \times 10^{-01}$ \\
\hline $\mathrm{PCl}$ & $136(16.3)$ & $32(21.9)$ & $1.20 \times 10^{-01}$ \\
\hline Chronic heart failure & $33(4.0)$ & $26(17.8)$ & $2.62 \times 10^{-10}$ \\
\hline Atrial fibrillation & $42(5.0)$ & $21(14.4)$ & $4.60 \times 10^{-05}$ \\
\hline Cerebrovascular disease & $50(6.0)$ & $28(19.2)$ & $1.28 \times 10^{-07}$ \\
\hline \multicolumn{4}{|l|}{ Presentation features } \\
\hline ACS classification, STEMI, n (\%) & $343(41.2)$ & $35(24.0)$ & $1.15 \times 10^{-04}$ \\
\hline Killip II-IV, n (\%) & $114(13.7)$ & $61(41.8)$ & $6.33 \times 10^{-16}$ \\
\hline Creatinine $>1.2 \mathrm{mg} / \mathrm{dL}, n(\%)$ & $129(15.5)$ & $54(37.0)$ & $4.08 \times 10^{-09}$ \\
\hline Heart rate (bpm), median (IQR) & $80(67-91)$ & $90(80-105)$ & $3.66 \times 10^{-06}$ \\
\hline Positive peak troponine maximum, $n(\%)$ & $804(96.6)$ & $143(97.9)$ & $6.98 \times 10^{-01}$ \\
\hline Positive peak CK-MB maximum, $n(\%)$ & $422(50.7)$ & $50(32.2)$ & $1.88 \times 10^{-03}$ \\
\hline GRACE score $>118, n(\%)$ & $687(82.6)$ & $123(84.2)$ & $1.62 \times 10^{-01}$ \\
\hline
\end{tabular}

CAD coronary artery diseases, CABG coronary artery bypass grafting, $P C l$ percutaneous coronary intervention, $A C S$ acute coronary syndrome, STEMI ST-segment elevation myocardial infarction, CK-MB creatine kinase-MB, GRACE Global Registry of Acute Coronary Events

Spectra of the validation samples were classified as either 'dead' or 'survivor' using the optimized NOESY RF risk score model derived from the training set. Comparison between metabolomic classification and actual outcome demonstrated high correlation with an AUC of 0.801 (Fig. 1c). Using the threshold maximized in the training set, we obtained $72.6 \%$ sensitivity, $72.6 \%$ specificity, and $72.6 \%$ overall predicting accuracy.

The AUC calculated on the RF score was assessed for significance against the null hypothesis of no prediction accuracy in the data by means of 10,000 randomized class-permutations tests: the estimated AUC obtained after randomization was 0.570 (95\% CI $0.569-0.571$ ), demonstrating the significance of our result (AUC 0.801, $\left.p=2.21 \times 10^{-06}\right)$.

The performances of the NOESY RF model in the validation set was assessed using a Cox proportional hazards regression model: NOESY RF showed a hazard ratio of $6.16(95 \%$ CI 4.02-9.44) with a $p$-value of $1.11 \times 10^{-16}$ calculated using the likelihood-ratio test, and a concordance coefficient of 0.71 .

\section{Comparison of the NOESY RF score with known prognostic factors and the GRACE score}

The known prognostic factors age, sex, previous CABG, previous PCI, heart failure, atrial fibrillation, cerebrovascular disease, diabetes, creatinine concentration, Killip class, and ACS classification were compared with the NOESY RF risk score, calculated on the entire dataset, in univariate and multivariate Cox regression analyses. The results are displayed in Table 2 .

In the univariate analysis, many prognostic factors were statistically associated with the outcomes, among them the NOESY RF score showed a Cox hazard ratio of 6.45. In the multivariate analysis, only age, dyslipidemia, hypertension, previous cerebrovascular disease, Killip 


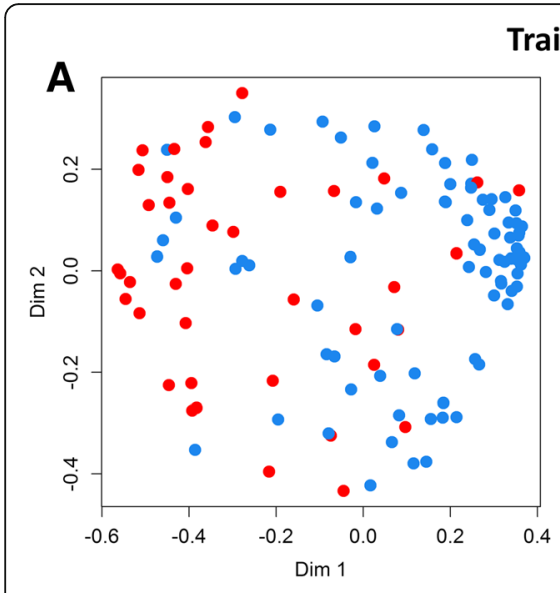

Accuracy NOESY model: $\mathbf{7 8 . 0 \%}$
Training set

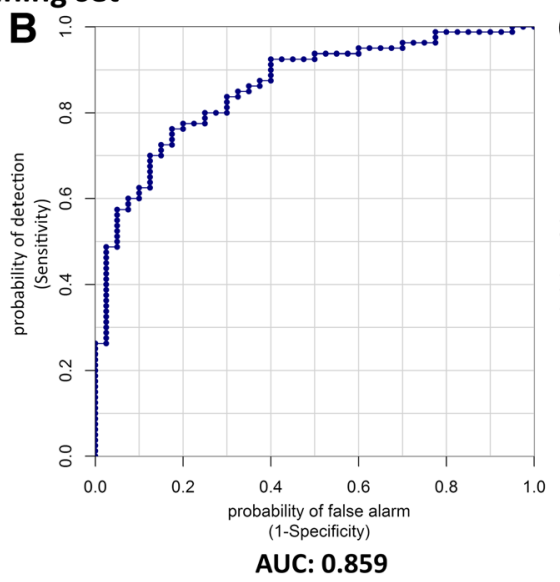

Validation set

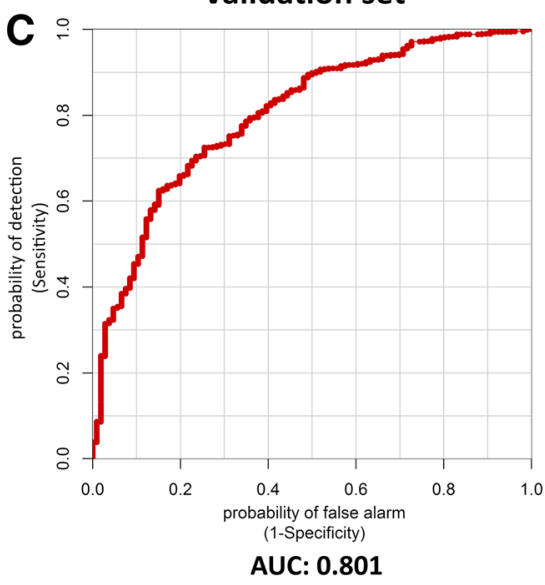

Fig. 1 Clusterization of serum metabolomic profiles and comparisons between metabolomic classification and outcomes in the training set and the validation set. a Discrimination between patients who survived (blue dots, $\mathrm{n}=80$ ) and died (red dots, $\mathrm{n}=40$ ) using the Random Forest classifier on nuclear Overhauser effect spectroscopy (NOESY) spectra in the training set. b, $\mathbf{c}$ The receiver operator characteristic curves and the area under the curve (AUC) scores are presented for the training set (b) and validation set (c)

class, and NOESY RF score remained statistically significant, demonstrating their independence with respect to the other variables. The smoker's paradox, already described by other studies [39], was also observed in this cohort.

The performance of the NOESY RF score in predicting 2-year outcomes was also compared with the performance of the GRACE hospital discharge risk score [40] for those patients for whom all clinical parameters needed to calculate the GRACE score were available $(84.5 \%$ of patients). The results (Harrell's c index and AUC) demonstrated that the NOESY RF score performed slightly better in both the training and the validation sets (Table 3, Fig. 2), confirming that the metabolomic approach described here could be useful for risk stratification in the setting of post-AMI patients. Moreover, the score obtained by a linear combination of the two scores (NOESY RF + GRACE scores) was calculated (Table 3, Fig. 2); the combined model showed statistically improved results (analysis of variance test $p$-value $<0.01$ after Bonferroni correction) with respect to the two separate scores in both the training and validation sets. A comparison of these two scores with the NOESY RF score is included in the Cox regression analyses in Table 2.

Using ROC analyses, the performance of the NOESY RF and GRACE scores was tested against some well-known parameters that are commonly measured in the clinical practice (age, heart frequency, diastolic pressure, systolic pressure, creatinine concentration, glycemia, platelets). Training and validation sets were considered separately and only data from patients for whom all the clinical parameters were available were included (80.5\% of the entire cohort). As shown in Fig. 3, our score showed the best performances in predicting death within 2 years from the cardiovascular event both in training and validation sets (AUC 0.837 and 0.797, respectively); age showed comparable results (AUC 0.828 and 0.747 ) and the GRACE score presented slightly worse results (AUC 0.809 and 0.75).

The performance of our approach was also tested by dividing patients in two distinct subgroups of patients according to AMI severity (ST-segment elevation myocardial infarction (STEMI) and non-STEMI). The results demonstrated that our approach is marginally affected by these two subcategories (Additional file 1: Supplementary material and Figure S6).

\section{Metabolites analysis}

An analysis of the NMR spectra was conducted to identify which metabolites were statistically different between patients who died and survived. All patients (training and validation sets) were included in the analysis. The following 23 metabolites were unambiguously assigned and quantified in the spectra: acetate, acetone, alanine, citrate, creatine, creatinine, formate, glucose, glutamate, glutamine, glycine, histidine, isobutyrate, isoleucine, lactate, leucine, mannose, methionine, phenylalanine, proline, tyrosine, valine, and 3-hydroxybutyrate. We observed that patients who died were characterized by significantly (adjusted $p$-value $<0.05$ ) higher levels of 3-hydroxybutyrate, proline, creatinine, acetate, acetone, formate, and mannose, and significantly lower levels of valine and histidine (Additional file 1: Table S2).

Metabolite analyses replicated separately in the training and validation sets are provided in Additional file 1: Figure S7. 
Table 2 Association with the outcome: unadjusted and adjusted hazard ratios

\begin{tabular}{|c|c|c|c|c|}
\hline & Hazard ratio (univariate) & $p$-value & Hazard ratio (multivariate) & $p$-value \\
\hline \multicolumn{5}{|l|}{ Age } \\
\hline $68-79$ & 4.63 & $2.24 \times 10^{-04}$ & 2.09 & $1.45 \times 10^{-01}$ \\
\hline$>79$ & 12.86 & $8.90 \times 10^{-11}$ & 3.87 & $8.33 \times 10^{-03}$ \\
\hline Male sex & 0.64 & $1.35 \times 10^{-02}$ & 0.96 & $8.72 \times 10^{-01}$ \\
\hline Hypertension & 1.34 & $1.47 \times 10^{-01}$ & 0.53 & $1.98 \times 10^{-02}$ \\
\hline Dyslipidemia & 0.55 & $7.16 \times 10^{-03}$ & 0.38 & $7.91 \times 10^{-03}$ \\
\hline \multicolumn{5}{|l|}{ Smoking habits } \\
\hline Yes & 0.43 & $1.67 \times 10^{-03}$ & 1.25 & $5.04 \times 10^{-01}$ \\
\hline Ex-smokers & 1.13 & $7.95 \times 10^{-01}$ & $4.14 \times 10^{-08}$ & $9.95 \times 10^{-01}$ \\
\hline CAD & 0.41 & $2.00 \times 10^{-03}$ & 0.99 & $9.86 \times 10^{-01}$ \\
\hline Previous CABG & 1.16 & $7.10 \times 10^{-01}$ & 1.92 & $2.61 \times 10^{-01}$ \\
\hline Previous PCl & 1.28 & $2.69 \times 10^{-01}$ & 1.66 & $9.64 \times 10^{-02}$ \\
\hline Heart failure & 3.67 & $1.07 \times 10^{-07}$ & 1.81 & $8.98 \times 10^{-02}$ \\
\hline Atrial fibrillation & 2.37 & $7.03 \times 10^{-04}$ & 1.30 & $4.52 \times 10^{-01}$ \\
\hline Cerebrovascular disease & 3.17 & $4.10 \times 10^{-07}$ & 1.96 & $2.67 \times 10^{-02}$ \\
\hline Diabetes & 1.99 & $1.83 \times 10^{-04}$ & 1.05 & $8.58 \times 10^{-01}$ \\
\hline Creatinine (>1.2 mg/dL) & 2.89 & $8.70 \times 10^{-09}$ & 1.29 & $3.37 \times 10^{-01}$ \\
\hline \multicolumn{5}{|l|}{ Killip class } \\
\hline$\|$ & 3.43 & $4.89 \times 10^{-09}$ & 1.77 & $4.26 \times 10^{-02}$ \\
\hline III & 4.87 & $9.12 \times 10^{-10}$ & 3.31 & $4.76 \times 10^{-04}$ \\
\hline \multicolumn{5}{|l|}{ ACS classification } \\
\hline STEMI & 0.49 & $1.34 \times 10^{-03}$ & 0.72 & $3.00 \times 10^{-01}$ \\
\hline \multicolumn{5}{|l|}{ NOESY RF risk score } \\
\hline$\geq 0.454$ & 6.45 & $2.16 \times 10^{-16}$ & 3.71 & $2.36 \times 10^{-05}$ \\
\hline \multicolumn{5}{|l|}{ GRACE score ${ }^{\#}$} \\
\hline$\geq 170$ & 6.05 & $3.76 \times 10^{-06}$ & - & - \\
\hline \multicolumn{5}{|l|}{ NOESY RF + GRACE } \\
\hline$\geq 7.7$ & 9.33 & $2.16 \times 10^{-16}$ & - & - \\
\hline
\end{tabular}

Correlation with the outcome for prognostic features and RF risk score in the full dataset, using univariate and multivariate analysis. Age split into tertiles. In the multivariate, hazard ratios of all the variable were included together in the analysis

$C A D$ coronary artery diseases, $C A B G$ coronary artery bypass grafting, $P C I$ percutaneous coronary intervention, $A C S$ acute coronary syndrome, STEMI ST-segment elevation myocardial infarction, NOESY nuclear Overhauser effect spectroscopy, RF random forest, GRACE Global Registry of Acute Coronary Events

"These variables were not included in the multivariate analysis due to the strong co-linearity between GRACE score and the other clinical variables [66]

Table 3 Comparison between GRACE score, NOESY RF score and a linear combination of NOESY RF and GRACE sCores (GRACE + NOESY RF scores)

\begin{tabular}{llll}
\hline & GRACE score & NOESY RF score & GRACE + NOESY RF sCores \\
\hline Training set & & & \\
$\quad$ AUC $(95 \% \mathrm{Cl})$ & $0.815(0.794-0.820)$ & $0.859(0.858-0.860)$ & $0.875(0.864-0.885)$ \\
$\quad$ Harrell's c index & $0.776(0.761-0.781)$ & $0.806(0.805-0.809)$ & $0.828(0.821-0.835)$ \\
Validation set, & $0.756(0.754-0.758)$ & $0.801(0.800-0.802)$ & $0.823(0.822-0.825)$ \\
AUC & $0.740(0.744-0.747)$ & $0.789(0.789-0.790)$ & $0.809(0.808-0.810)$ \\
$\quad$ Harrell's C index & & & \\
\hline
\end{tabular}

GRACE Global Registry of Acute Coronary Events, NOESY nuclear Overhauser effect spectroscopy, RF random forest, AUC area under the receiver operating characteristic, $\mathrm{Cl}$ confidence interval 

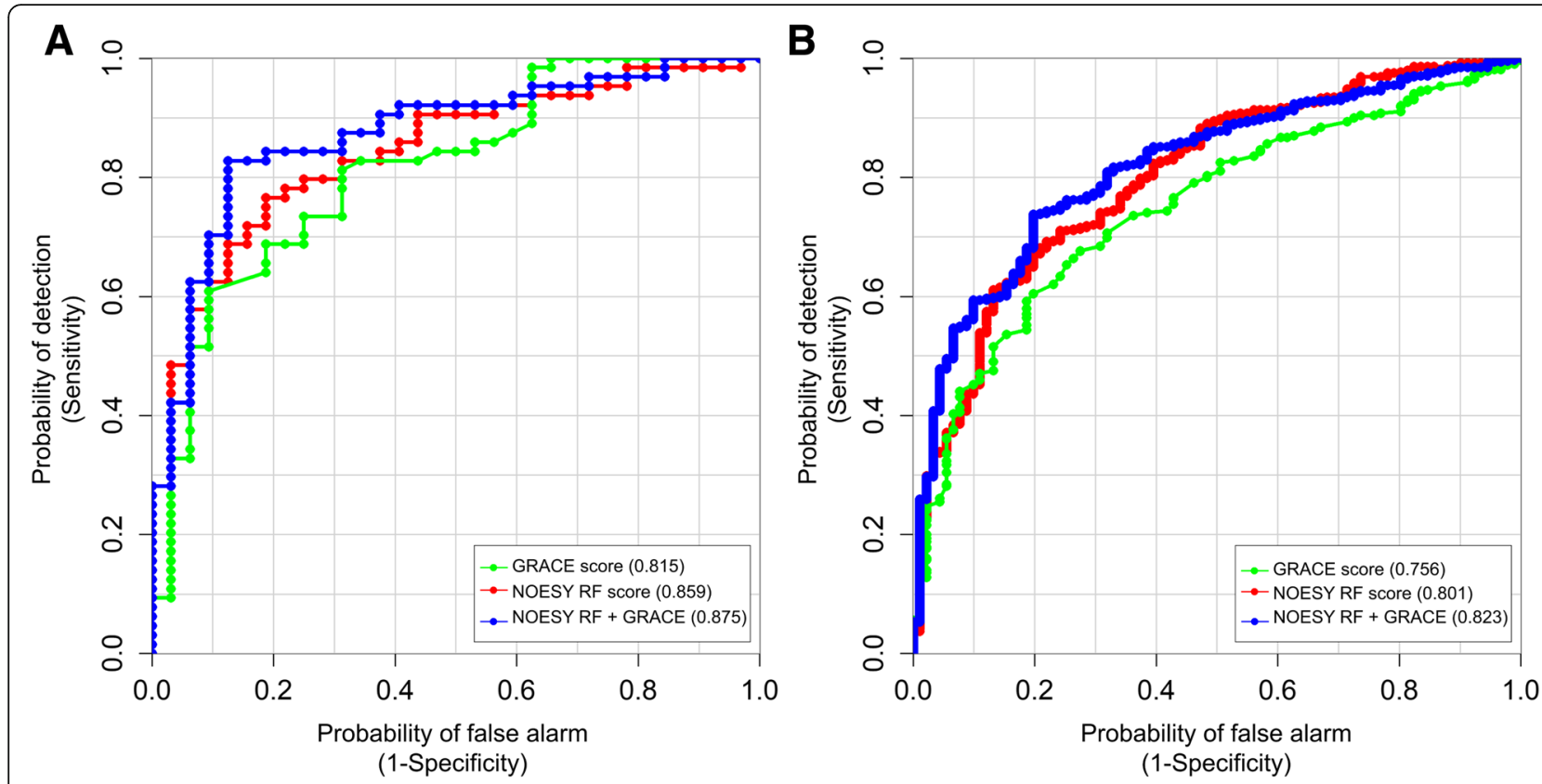

Fig. 2 Receiver operator characteristic curve and area under the curve (AUC) of nuclear Overhauser effect spectroscopy (NOESY) random forest (RF) score, Global Registry of Acute Coronary Events (GRACE) score, and linear combined score of NOESY RF and GRACE score are reported for the $\mathbf{a}$ training and $\mathbf{b}$ validation sets
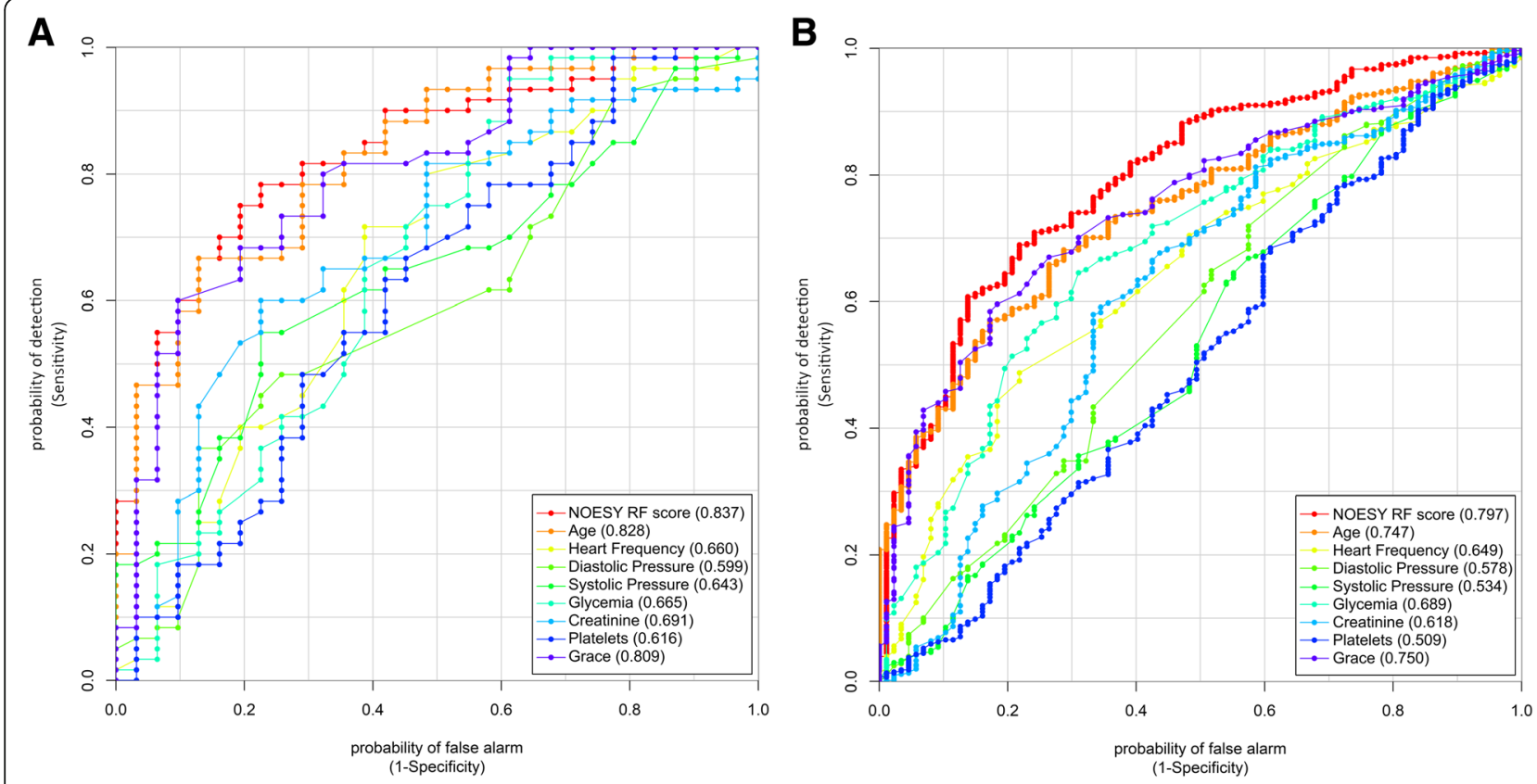

Fig. 3 Nuclear Overhauser effect spectroscopy (NOESY) random forest (RF) score, Global Registry of Acute Coronary Events (GRACE) score, and clinical parameters receiver operator characteristic (ROC) curves for the $\mathbf{a}$ training and $\mathbf{b}$ validation sets. The ROC curves and the area under the curve scores are presented for NOESY RF score, GRACE score, age, heart frequency, diastolic pressure, systolic pressure, glycemia, creatinine, platelets, troponine maximum, and creatine kinase-MB maximum 


\section{Sex-specific RF models}

The presence of sex-specific differences in human metabolism is well known and the NMR metabolomics is sensitive to these differences $[41,42]$. Therefore, we decided to build different RF models for women and men to obtain an outcome clusterization and prediction unbiased by sex.

For each sex-specific group using the RF classifier, the metabolomic NOESY one-dimensional profiles of 60 patients who survived and 30 who died were randomly selected for classification. Both sex models showed significant differential clustering, with a good separation of the two outcome groups (Additional file 1: Figure S8a, d).

Using the NOESY NMR spectra of the female and male cohorts separately, the RF classifier discriminated patients who died from those who survived in the training set with an AUC of 0.786 and 0.834 , respectively (Additional file 1: Figure S8b, e). Thresholds of $\geq 0.46$ for the female cohort and $\geq 0.476$ for the male cohort were set in the training set by optimizing accuracy, sensitivity, and specificity. The results for both cohorts are shown in Additional file 1: Table S3.

When applying these models to the validation set of female and male cohorts, we obtained an AUC of 0.782 and 0.821 , respectively (Fig. $3 \mathrm{c}, \mathrm{f}$ ). When using the threshold maximized in the training set, we obtained an overall accuracy of $74.7 \%$ and $73.4 \%$ for predicting the likelihood of outcome in female and male cohorts, respectively (Additional file 1: Table S3).

On analyzing the spectra of the female and male cohorts, we observed that female patients who died were characterized by higher levels of creatinine, whereas male patients who died were characterized by higher levels of 3-hydroxybutyrate, proline, creatinine, and formate, and significantly lower levels of histidine and valine (Additional file 1: Figure S7b, c).

\section{Discussion}

After the acute phase of an AMI, for which management is strictly defined by the European Society of Cardiology Guidelines [43, 44], patients remain at increased risk of secondary atherothrombotic events, including recurrent ACS events and stroke, and continue to face a high risk of premature death not only in the immediate future but also in the following years $[45,46]$. For these reasons, the identification of a metabolomic fingerprint able to identify patients who are at increased risk of death might allow clinicians to tailor medical treatments and interventions according to patients' overall risk: high-risk patients could be targeted with more intensive pharmacological treatments (that is, with the highest tolerated statin dosage or more aggressive antiplatelet treatments), and more intensive follow-up programs could be planned with clinical reevaluation at shorter time intervals (that is, monthly instead of the standard visits at 1 , 6, and 12 months). In AMI patients enrolled in the AMI-Florence 2 study, we found a metabolic fingerprint which was able to discriminate patients who died within 2 years from the cardiovascular event from survivors with high accuracy (AUC 0.859), and this result was duplicated in a validation set (AUC 0.801). We also built sex-specific RF models and found that the male model was better able to predict outcomes (male: AUC 0.834; female: AUC 0.786), which was confirmed in the validation set. To the best of our knowledge, this is the first study to assess the capability of a metabolomic assay to predict mortality in the setting of AMI.

A metabolic fingerprint can be deemed as a holistic super-biomarker with a discriminative and predictive power undoubtedly higher than that of the sum of the few quantified metabolites [47]. AMI, as with the majority of human diseases, has a multifactorial etiology and a complex physiopathology that concurrently alters several metabolic pathways [48]. Therefore, the metabolic fingerprint, composed by superimposing all the visible signals of the low and high molecular weight endogenous metabolites, represents an optimal level at which to analyze pathological changes in biological systems [49]; indeed, it takes into account all metabolite variations, even slight ones.

The NOESY RF score, based on the metabolic fingerprint here presented, was independent from the classical clinical parameters and the widely used GRACE score, and achieved better results in predicting all-cause death within 2 years after AMI when considering both Cox models and ROC analyses. It is worth of mentioning that age was a very good predictor of mortality in our dataset; in particular, it proved to be better than even the GRACE score in our training set. Older patients showed the worst clinical conditions, with a higher percentage of heart failure, atrial fibrillation, previous cerebrovascular diseases, and diabetes. These parameters are not included in the GRACE score model, and could explain why age performed very well as a predictor in our cohort.

Although our study has several strengths, including the number of patients studied, the long-term follow-up ( 2 years), and the analysis replication in a validation set, some limitations should also be mentioned. First, sample collections were done exclusively in the acute phase of the disease, impairing the acquisition of data correlated to the biochemical mechanisms of the transition to the quiescent phase. Owing to the importance of this aspect, further efforts in this direction are required. Second, even though the data were replicated in a validation set, a totally independent cohort for validation is lacking. However, before attempting to replicate these findings in very large multicenter studies, common standard 
operating procedures are required for sample collection and storage, otherwise samples collected in different centers will not be comparable. Our group is strongly committed to this, and have contributed to the development of the optimal pre-analytical procedures for metabolomics [25]. Finally, NMR is less sensitive than MS (although it is more suitable for metabolic fingerprinting), and thus only a limited number of metabolites have been found to be statistically significant. Specifically, we found that patients who died were characterized by significantly higher levels of 3-hydroxybutyrate, proline, creatinine, acetate, acetone, formate, and mannose, and significantly lower levels of valine and histidine.

Lifestyle and the medication administered to a patient influence the molecular signatures in plasma and serum samples, and the relative metabolite concentrations reflect tissue lesions and organ dysfunctions. In this framework, previous studies have underlined the usefulness of metabolomics of serum and plasma in determining the individual's disease risk, prognosis, and therapeutic options in different clinical settings [29, 30, 50, 51]. For instance, sera of heart failure patients carries a strong signature of the disease, allowing the estimation of heart failure-related metabolic disturbance and possessing a better prognostic value than conventional biomarkers $[13,51]$.

In a prospective study of three population-based cohorts from Finland that were free of CVD at baseline [52], a metabolomic analysis evidenced that circulating phenylalanine, monounsaturated fats, and polyunsaturated fatty acids were as strongly predictive of cardiovascular risk as the conventional lipid risk factors, and were markers of CVD onset during a long-term follow-up (more than a decade). In our study, we did not find any role for these metabolites in predicting mortality. However, the different study populations (CVD- free vs. AMI patients) with different lifestyle and dietary habits may explain the different molecular signatures.

The usefulness of the metabolomic approach was also demonstrated in a general cohort of patients at risk for cardiovascular events undergoing cardiac catheterization [53]; at baseline, plasma metabolomic profiles independently predicted cardiovascular death after adjustment for multiple clinical covariates. In this study, a significant predictive role was demonstrated for five metabolite factors (medium-chain acylcarnitines, short-chain dicarboxylacylcarnitines, long-chain dicarboxylacylcarnitines, branched-chain amino acids, and fatty acids). We consistently found in the present study that higher levels of valine were a protective factor in AMI patients. At variance with our study, increased concentrations of branched-chain amino acids levels have been shown in coronary artery patients compared with control subjects [54], and high levels of these essential amino acids significantly correlated with the severity of coronary artery disease (CAD) [55]. However, these studies were case-control studies and did not evaluate CAD patients in the acute phase of the disease. The pathways of the branched amino acids in humans are very complex and it is likely that in ACS patients an altered metabolic pathway for these amino acids represents a prognostic risk factor.

Our study, performed in a large sample population of AMI patients followed for 2 years, provided new data about the role of high 3-hydroxybutyrate circulating levels on post-AMI mortality. A previous study that evaluated the ketone bodies in the urine of five ACS patients demonstrated that ketone bodies, and particularly 3-hydroxybutyrate, were altered during the acute event [56]. Furthermore, high levels of 3-hydroxybutyrate have been associated with high prevalence of heart failure and diabetes [57]. In insulin deficiency, when the release of free fatty acids from adipose tissues exceeds the capacity of the tissues to metabolize them, severe and potentially fatal diabetic ketoacidosis can occur in which levels of 3-hydroxybutyrate in the blood can reach up to $25 \mathrm{mM}$ [58]. A recent study has also demonstrated a significant increase of serum ketone bodies in response to angioplasty-induced ischemia performed in patients with stable angina, and it has been hypothesized that these metabolic changes could be a response to reperfusion oxidative stress and may play a key role in free radical homeostasis during ischemia-reperfusion injury [59]. However, it remains unclear whether the elevation of ketone bodies represents an adaptive mechanism required to maintain cell metabolism or if it actually contributes to disease progression and, thus, the worsening of the prognosis.

Furthermore, it is worth of mentioning that formate has already been proposed as a possible biomarker of ACS [60].

As expected, patients with worst prognosis showed higher level of serum creatinine, a well-known marker of renal insufficiency. It has already been demonstrated that elevated serum levels of creatinine on admission are associated with impaired myocardial flow and poor prognosis for 1-year mortality [61, 62].

Previous studies evidenced an interaction between sex and adverse cardiovascular events in CAD patients [6365], with myocardial infarction morbidity and mortality higher in women than in men. Consistently, our results demonstrated a higher prevalence of mortality in women than in men (19.4\% vs. $12.5 \%)$. The excess risk of mortality in women could be ascribed to the differences in cardiovascular risk factor, that is, age, hypertension, diabetes, and co-morbidities prevalence. Accordingly, in our study, women showed a higher median age and higher prevalence of hypertension, atrial fibrillation, and heart failure with respect to men. 
Building sex-specific models enabled us to improve the outcome prediction in the male cohort, but not in the female one. Thus, the male metabolic fingerprint seems to show a higher association with cardiovascular mortality than the female one. However, the female cohort was smaller than the male cohort and this may have affected the predictive capability of the model; therefore, larger cohorts of patients are needed to build robust sex-specific models.

\section{Conclusions}

Our data support the usefulness of the metabolomic approach for identifying a more precise risk profile in AMI patients. Metabolomic analysis by NMR enables fast, approachable, and reproducible characterization of the AMI metabolic fingerprint associated with a poor prognosis, improving the cardiovascular risk assessment beyond that achieved by established risk factors, and identifying those patients who need to undergo a very early and aggressive treatment. Thus, metabolomics could represent a valid addition to the already established risk stratification tools. Further, as demonstrated by the combination of the NOESY RF scores with the Grace score, metabolomics, in combination with classical tests, is able to significantly improve risk classification over the two separate scores.

\section{Additional file}

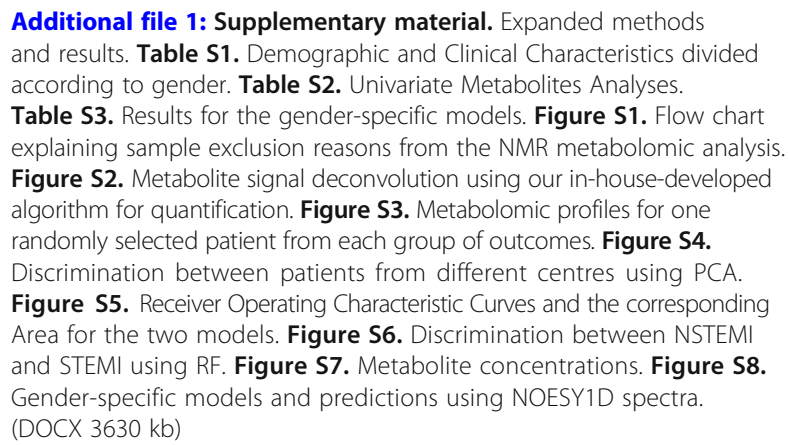

\section{Abbreviations}

ACS: Acute coronary syndrome; AMI: Acute myocardial infarction; AUC: Area under the curve; CAD: Coronary artery disease; Cl: Confidence interval; CPMG: Carr-Purcell-Meiboom-Gill; CVDs: Cardiovascular diseases; GRACE: Global Registry of Acute Coronary Events; MS: Mass spectrometry; NMR: Nuclear magnetic resonance; NOESY: Nuclear Overhauser effect spectroscopy; PCl: Percutaneous coronary intervention; RF: Random forest; ROC: Receiver operating characteristics; STEMI: ST-segment elevation myocardial infarction

\section{Acknowledgements}

The authors acknowledge the support and the use of resources of Instruct-ERIC, a Landmark ESFRI project, and specifically the CERM/CIRMMP Italy Centre.

\section{Funding}

The NMR analysis of this study was partially supported by the European Commission projects Phenomenal (contract no. 654241) and Propag-ageing (contract no. 634821). The authors acknowledge the Regione Toscana that partially founded the project "AMI-Florence 2: coronary reperfusion and other invasive cardiology procedures in the treatment of acute myocardial infarction and acute coronary syndromes in the Florentine area".

\section{Availability of data and materials}

NMR and clinical data are freely available in the Open-Access Database Repository MetaboLights from October 2017 with the accession number MTBLS395 (http://www.ebi.ac.uk/metabolights).

\section{Authors' contributions}

$\mathrm{BG}, \mathrm{RM}, \mathrm{CL}$, and $\mathrm{AMG}$ designed the study. BG, SV, NC, DB, AB, NM, GFG, RM, and $A M G$ recruited patients for the study and collected serum samples. AV, $\mathrm{LT}$, and PGT collected NMR data and performed statistical analyses. AV, LT, $B G, A M G$, and $C L$ interpreted the data and wrote the manuscript. All authors had full access to all of the data in the study and take responsibility for the integrity of the data and the accuracy of the data analysis. All authors read and approved the final manuscript.

Ethics approval and consent to participate

All subjects gave written informed consent. The study (number 11/2008) complies with the Declaration of Helsinki and was approved by the ethics committees of the local health unit, the University of Florence, and Careggi Hospital (19 March 2008).

\section{Consent for publication}

Not applicable

\section{Competing interests}

The authors declare that they have no competing interests.

\section{Publisher's Note}

Springer Nature remains neutral with regard to jurisdictional claims in published maps and institutional affiliations.

\section{Author details}

${ }^{1}$ Magnetic Resonance Center (CERM), University of Florence, Sesto Fiorentino, Italy. ${ }^{2}$ Consorzio Interuniversitario Risonanze Magnetiche di Metallo Proteine C.I.R.M.M.P, Sesto Fiorentino, Italy. ${ }^{3}$ Department of Experimental and Clinical Medicine, University of Florence, Florence, Italy. ${ }^{4}$ Careggi Hospital, Florence, Italy. ${ }^{5}$ Giotto Biotech S.r.l, Sesto Fiorentino, Florence, Italy. ${ }^{6}$ Unit of Epidemiology, ASL 10, Florence, Italy. ${ }^{7}$ Centro Studi Medicina Avanzata (CESMAV), Florence, Italy. ${ }^{8}$ Department of Chemistry, University of Florence, Sesto Fiorentino, Italy.

Received: 27 December 2017 Accepted: 14 December 2018 Published online: 07 January 2019

\section{References}

1. Wright RS, Anderson $J \mathrm{~L}$, Adams CD, Bridges CR, Casey DE, Ettinger SM, et al. 2011 ACCF/AHA focused update incorporated into the ACC/AHA 2007 Guidelines for the management of patients with unstable angina/non-stelevation myocardial infarction: a report of the American College of Cardiology Foundation/American Heart Association Task Force on Practice Guidelines developed in collaboration with the American Academy of Family Physicians, Society for Cardiovascular Angiography and Interventions, and the Society of Thoracic Surgeons. J Am Coll Cardiol. 2011;57:e215-367.

2. Mozaffarian D, Benjamin EJ, Go AS, Arnett DK, Blaha MJ, Cushman M, et al. Heart disease and stroke statistics--2015 update: a report from the American Heart Association. Circulation. 2015;131:e29-322.

3. Granger CB, Goldberg RJ, Dabbous O, Pieper KS, Eagle KA, Cannon CP, et al. Predictors of hospital mortality in the global registry of acute coronary events. Arch Intern Med. 2003;163:2345-53.

4. Antman EM, Cohen M, Bernink PJ, McCabe CH, Horacek T, Papuchis G, et al. The TIMI risk score for unstable angina/non-ST elevation Ml: a method for prognostication and therapeutic decision making. JAMA. 2000;284:835-42.

5. Cavusoglu E, Marmur JD, Yanamadala S, Chopra V, Hegde S, Nazli A, et al. Elevated baseline plasma IL-8 levels are an independent predictor of longterm all-cause mortality in patients with acute coronary syndrome. Atherosclerosis. 2015;242:589-94. 
6. Emerging Risk Factors Collaboration, Kaptoge S, Di Angelantonio E, Lowe G, Pepys MB, Thompson SG, et al. C-reactive protein concentration and risk of coronary heart disease, stroke, and mortality: an individual participant metaanalysis. Lancet. 2010;375:132-40.

7. Vignoli A, Ghini V, Meoni G, Licari C, Takis PG, Tenori L, et al. Highthroughput metabolomics by 1D NMR. Angew Chem Int Ed Engl. 2018. https://doi.org/10.1002/anie.201804736.

8. Nicholson JK, Lindon JC. Systems biology: metabonomics. Nature. 2008;455: 1054-6

9. DeFilippis AP, Trainor PJ, Hill BG, Amraotkar AR, Rai SN, Hirsch GA, et al. Identification of a plasma metabolomic signature of thrombotic myocardial infarction that is distinct from non-thrombotic myocardial infarction and stable coronary artery disease. PloS One. 2017;12:e0175591.

10. Gallo V, Intini N, Mastrorilli P, Latronico M, Scapicchio P, Triggiani M, et al. Performance assessment in fingerprinting and multi component quantitative NMR analyses. Anal Chem. 2015;87:6709-17.

11. Eckhart AD, Beebe K, Milburn M. Metabolomics as a key integrator for "omic" advancement of personalized medicine and future therapies. Clin Transl Sci. 2012:5:285-8.

12. Zordoky BN, Sung MM, Ezekowitz J, Mandal R, Han B, Bjorndahl TC, et al. Metabolomic fingerprint of heart failure with preserved ejection fraction. PloS One. 2015;10:e0124844.

13. Tenori L, Hu X, Pantaleo P, Alterini B, Castelli G, Olivotto I, et al. Metabolomic fingerprint of heart failure in humans: a nuclear magnetic resonance spectroscopy analysis. Int J Cardiol. 2013;168:e113-5.

14. Delles C, Rankin NJ, Boachie C, McConnachie A, Ford I, Kangas A, et al. Nuclear magnetic resonance-based metabolomics identifies phenylalanine as a novel predictor of incident heart failure hospitalisation: results from PROSPER and FINRISK 1997. Eur J Heart Fail. 2018;20:663-73.

15. Alonso A, Yu B, Qureshi WT, Grams ME, Selvin E, Soliman EZ, et al. Metabolomics and incidence of atrial fibrillation in African Americans: the Atherosclerosis Risk in Communities (ARIC) study. PloS One. 2015;10:e0142610.

16. Liu X, Gao J, Chen J, Wang Z, Shi Q, Man H, et al. Identification of metabolic biomarkers in patients with type 2 diabetic coronary heart diseases based on metabolomic approach. Sci Rep. 2016;6:30785.

17. Bodi V, Sanchis J, Morales JM, Marrachelli VG, Nunez J, Forteza MJ, et al. Metabolomic profile of human myocardial ischemia by nuclear magnetic resonance spectroscopy of peripheral blood serum: a translational study based on transient coronary occlusion models. J Am Coll Cardiol. 2012;59:1629-41.

18. Ali SE, Farag MA, Holvoet P, Hanafi RS, Gad MZ. A comparative metabolomics approach reveals early biomarkers for metabolic response to acute myocardial infarction. Sci Rep. 2016;6:36359.

19. Trainor PJ, Yampolskiy RV, DeFilippis AP. Wisdom of artificial crowds feature selection in untargeted metabolomics: an application to the development of a blood-based diagnostic test for thrombotic myocardial infarction. J Biomed Inform. 2018;81:53-60.

20. Yin X, de Carvalho LP, Chan MY, Li SFY. Integrated metabolomics and metallomics analyses in acute coronary syndrome patients. Met Integr Biometal Sci. 2017;9:734-43.

21. Park JY, Lee S-H, Shin M-J, Hwang G-S. Alteration in metabolic signature and lipid metabolism in patients with angina pectoris and myocardial infarction. PloS One. 2015;10:e0135228.

22. von Zur MC, Schiffer E, Zuerbig $P$, Kellmann $M$, Brasse $M$, Meert $N$, et al. Evaluation of urine proteome pattern analysis for its potential to reflect coronary artery atherosclerosis in symptomatic patients. J Proteome Res. 2009:8:335-45.

23. Cesari F, Marcucci R, Gori AM, Caporale R, Fanelli A, Casola G, et al. Reticulated platelets predict cardiovascular death in acute coronary syndrome patients. Insights from the AMI-Florence 2 Study. Thromb Haemost. 2013;109:846-53.

24. Cohen J. Statistical power analysis for the behavioral sciences. L. Erlbaum Associates: Michigan; 1988

25. Bernini P, Bertini I, Luchinat C, Nincheri P, Staderini S, Turano P. Standard operating procedures for pre-analytical handling of blood and urine for metabolomic studies and biobanks. J Biomol NMR. 2011;49:231-43.

26. Beckonert $\mathrm{O}$, Keun HC, Ebbels TMD, Bundy J, Holmes E, Lindon JC, et al. Metabolic profiling, metabolomic and metabonomic procedures for NMR spectroscopy of urine, plasma, serum and tissue extracts. Nat Protoc. 2007;2: 2692-703.

27. Kotsiantis S, Kanellopoulos D, Pintelas P. Handling imbalanced datasets: a review. GESTS Int Trans Comp Sci Eng. 2005;30:25-36.
28. Breiman L. Random forests. Mach Learn. 2001;45:5-32.

29. Tenori L, Oakman C, Morris PG, Gralka E, Turner N, Cappadona S, et al. Serum metabolomic profiles evaluated after surgery may identify patients with oestrogen receptor negative early breast cancer at increased risk of disease recurrence. Results from a retrospective study. Mol Oncol. 2015;9:128-39.

30. Hart CD, Vignoli A, Tenori L, Uy GL, Van To T, Adebamowo C, et al. Serum metabolomic profiles identify ER-positive early breast cancer patients at increased risk of disease recurrence in a multicenter population. Clin Cancer Res. 2017:23:1422-31.

31. Chen T, Cao Y, Zhang Y, Liu J, Bao Y, Wang C, et al. Random forest in clinical metabolomics for phenotypic discrimination and biomarker selection. Evid-Based Complement Altern Med. 2013;2013:298183.

32. Liaw A, Wiener M. Classification and regression by random forest. R News. 2002;2:18-22.

33. Cox DR. Regression models and life-tables. In: Kotz S, Johnson NL, editors. Breakthroughs in statistics. New York: Springer; 1992. p. 527-41.

34. Psychogios N, Hau DD, Peng J, Guo AC, Mandal R, Bouatra S, et al. The human serum metabolome. PLoS One. 2011;6:e16957.

35. Wishart DS, Jewison T, Guo AC, Wilson M, Knox C, Liu Y, et al. HMDB 3.0-The Human Metabolome Database in 2013. Nucleic Acids Res. 2013;41: D801-7.

36. Wilcoxon F. Individual Comparisons by ranking methods. Biom Bull. 1945;1:80.

37. Benjamini $Y$, Hochberg $Y$. Controlling the false discovery rate: a practical and powerful approach to multiple testing. J R Stat Soc Ser B Methodol. 1995;1:289-300.

38. Cliff N. Ordinal methods for behavioral data analysis. Mahwah: Psychology Press; 1996.

39. Robertson JO, Ebrahimi R, Lansky AJ, Mehran R, Stone GW, Lincoff AM Impact of cigarette smoking on extent of coronary artery disease and prognosis of patients with non-ST-segment elevation acute coronary syndromes: an analysis from the ACUITY trial (Acute Catheterization and Urgent Intervention Triage Strategy). JACC Cardiovasc Interv. 2014;7:372-9.

40. Tang EW, Wong C-K, Herbison P. Global Registry of Acute Coronary Events (GRACE) hospital discharge risk score accurately predicts long-term mortality post acute coronary syndrome. Am Heart J. 2007;153:29-35.

41. Kochhar S, Jacobs DM, Ramadan Z, Berruex F, Fuerholz A, Fay LB. Probing gender-specific metabolism differences in humans by nuclear magnetic resonance-based metabonomics. Anal Biochem. 2006:352:274-81.

42. Vignoli A, Tenori L, Luchinat C, Saccenti E. Age and sex effects on plasma metabolite association networks in healthy subjects. J Proteome Res. 2018; 17:97-107.

43. Ibanez B, James S, Agewall S, Antunes MJ, Bucciarelli-Ducci C, Bueno H, et al. 2017 ESC guidelines for the management of acute myocardial infarction in patients presenting with ST-segment elevation: the Task Force for the management of acute myocardial infarction in patients presenting with ST-segment elevation of the European Society of Cardiology (ESC). Eur Heart J. 2018:39:119-77.

44. Roffi M, Patrono C, Collet J-P, Mueller C, Valgimigli M, Andreotti F, et al. 2015 ESC guidelines for the management of acute coronary syndromes in patients presenting without persistent ST-segment elevation: Task Force for the management of acute coronary syndromes in patients presenting without persistent ST-segment elevation of the European Society of Cardiology (ESC). Eur Heart J. 2016;37:267-315.

45. Atar D, Bode C, Stuerzenbecher A, Verheugt FWA. Anticoagulants for secondary prevention after acute myocardial infarction: lessons from the past decade. Fundam Clin Pharmacol. 2014;28:353-63.

46. Bhatt DL, Eagle KA, Ohman EM, Hirsch AT, Goto S, Mahoney EM, et al. Comparative determinants of 4-year cardiovascular event rates in stable outpatients at risk of or with atherothrombosis. JAMA. 2010;304:1350-7.

47. Vignoli A, Rodio DM, Bellizzi A, Sobolev AP, Anzivino E, Mischitelli M, et al. NMR-based metabolomic approach to study urine samples of chronic inflammatory rheumatic disease patients. Anal Bioanal Chem. 2017;409: 1405-13.

48. Nicholson JK, Holmes E, Wilson ID. Gut microorganisms, mammalian metabolism and personalized health care. Nat Rev Microbiol. 2005;3:431-8.

49. Li J, Brazhnik O, Kamal A, Guo D, Lee C, Hoops S, et al. Metabolic profiling its role in biomarker discovery and gene function analysis. In: Harrigan G, editor. Goodacre R, editors. Dordrecht: Kluwer Academic; 2003. p. 293-309.

50. Basak T, Varshney S, Hamid Z, Ghosh S, Seth S, Sengupta S. Identification of metabolic markers in coronary artery disease using an untargeted LC-MS based metabolomic approach. J Proteomics. 2015;127:169-77. 
51. Cheng M-L, Wang C-H, Shiao M-S, Liu M-H, Huang Y-Y, Huang C-Y, et al. Metabolic disturbances identified in plasma are associated with outcomes in patients with heart failure: diagnostic and prognostic value of metabolomics. J Am Coll Cardiol. 2015;65:1509-20.

52. Würtz $P$, Havulinna AS, Soininen $P$, Tynkkynen T, Prieto-Merino $D$, Tillin $T$, et al. Metabolite profiling and cardiovascular event risk: a prospective study of 3 population-based cohorts. Circulation. 2015;131:774-85.

53. Shah SH, Sun J-L, Stevens RD, Bain JR, Muehlbauer MJ, Pieper KS, et al. Baseline metabolomic profiles predict cardiovascular events in patients at risk for coronary artery disease. Am Heart J. 2012;163:844-50.e1.

54. Yang RY, Wang SM, Sun L, Liu JM, Li HX, Sui XF, et al. Association of branched-chain amino acids with coronary artery disease: a matched-pair case-control study. Nutr Metab Cardiovasc Dis. 2015;25:937-42.

55. Bhattacharya S, Granger CB, Craig D, Haynes C, Bain J, Stevens RD, et al. Validation of the association between a branched chain amino acid metabolite profile and extremes of coronary artery disease in patients referred for cardiac catheterization. Atherosclerosis. 2014;232:191-6.

56. Martin-Lorenzo M, Zubiri I, Maroto AS, Gonzalez-Calero L, Posada-Ayala M, de la Cuesta F, et al. KLK1 and ZG16B proteins and arginine-proline metabolism identified as novel targets to monitor atherosclerosis, acute coronary syndrome and recovery. Metabolomics. 2015;11:1056-67.

57. Soininen P, Kangas AJ, Würtz P, Suna T, Ala-Korpela M. Quantitative serum nuclear magnetic resonance metabolomics in cardiovascular epidemiology and genetics. Circ Cardiovasc Genet. 2015;8:192-206.

58. Cahill GF, Veech RL. Ketoacids? Good medicine? Trans Am Clin Climatol Assoc. 2003:114:149-61 discussion 162-3.

59. Di Marino S, Viceconte N, Lembo A, Summa V, Tanzilli G, Raparelli V, et al. Early metabolic response to acute myocardial ischaemia in patients undergoing elective coronary angioplasty. Open Heart. 2018;5:e000709.

60. Carcía DD, Barba VI, MJJ A, Cristóbal VC. Diagnostic marker for acute coronary syndrome. Eur Patent Application. 2012; https://patents.google. com/patent/EP2535711A1/en.

61. Zhao L, Wang L, Zhang Y. Elevated admission serum creatinine predicts poor myocardial blood flow and one-year mortality in ST-segment elevation myocardial infarction patients undergoing primary percutaneous coronary intervention. J Invasive Cardiol. 2009;21:493-8.

62. Cakar MA, Gunduz H, Vatan MB, Kocayigit I, Akdemir R. The effect of admission creatinine levels on one-year mortality in acute myocardial infarction. Sci World J. 2012;2012:e186495.

63. Vaccarino V, Abramson JL, Veledar E, Weintraub WS. Sex differences in hospital mortality after coronary artery bypass surgery: evidence for a higher mortality in younger women. Circulation. 2002;105:1176-81.

64. Löwel H, Meisinger C, Heier M, Hörmann A, Kuch B, Gostomzyk J, et al. Sex specific trends of sudden cardiac death and acute myocardial infarction: results of the population-based KORA/MONICA-Augsburg register 1985 to 1998. Dtsch Med Wochenschr 1946. 2002;127:2311-6

65. Kunadian V, Qiu W, Lagerqvist B, Johnston N, Sinclair H, Tan Y, et al. Gender differences in outcomes and predictors of all-cause mortality after percutaneous coronary intervention (data from United Kingdom and Sweden). Am J Cardiol. 2017:119:210-6.

66. Vatcheva KP, Lee M, McCormick JB, Rahbar MH. Multicollinearity in regression analyses conducted in epidemiologic studies. Epidemiology (Sunnyvale). 2016. https://doi.org/10.4172/2161-1165.1000227.

Ready to submit your research? Choose BMC and benefit from:

- fast, convenient online submission

- thorough peer review by experienced researchers in your field

- rapid publication on acceptance

- support for research data, including large and complex data types

- gold Open Access which fosters wider collaboration and increased citations

- maximum visibility for your research: over $100 \mathrm{M}$ website views per year

At BMC, research is always in progress.

Learn more biomedcentral.com/submissions 